\title{
Input variables in bisensory memory
}

RHEA L. DORNBUSH ${ }^{1,2,3}$ DOUGLASS COLLEGE, RUTGERS-THE STATE UNIVERSITY

The effects of a combination of variables previously studied in ordinary and dichotic immediate memory were examined in bisensory memory, wherein the visual and auditory modalities were employed. These variables, combined in a factorial design. were: (1) type of material; (2) rate of presentation of material; (3) order of recall for a modality, either first or second; (4) locus of instructions about order of report; and (5) overall differences in memory for each modality. The variables of instruction, order of report, and nature of materials yielded effects in the bisensory situation similar to those reported for the dichotic and ordinary immediate memory situations. On the other hand, interference effects due to the combination of materials in the two channels were practically nonexistent. Additionally, when the modalities were stimulated simultaneously each displayed curves predictable from data obtained when it was explored independently of the other modality.

The present study is concerned with the effects of several variables on bisensory immediate memory in the visual and auditory modalities. In the bisensory experimental situation, different materials are simultaneously presented in two sensory modalities; $S$ is then required to recall all of the material from one modality followed by the material from the other modality.

One of the problems suggested by the simultaneous stimulation (bisensory) situation is that of division of attention. Specifically, does the individual have the ability to perform two simultaneous perceptual and/or memory tasks? The data thus far collected indicate that division of attention is possible, though not equally between two input channels. One's ability to divide attention between different sets of materials arriving along two channels is dependent on: order of report, instructions, rate of presentation, nature of materials, and channels involved. Thus, the effects of a combination of these variables were examined in bisensory memory, where the visual and auditory modalities were employed. More specifically, these variables were: (1) types of material, letters and/or numbers; (2) rate of presentation of material, one presentation 1/.5 sec (fast), 1/sec (medium), 1/2 sec (slow) (rates most often employed in short-term memory studies of this nature [Broadbent, 1954, 1956; Mackworth, 1962; Moray, 1960]); (3) order of recall for a modality, either first or second-this is specified in instructions accompanying each bisensory stimulus; (4) locus of instructions about order of report, either before or after presentation of the material; and (5) overall differences in memory for the visual and auditory modalities.

Each of the preceding variables has been shown to affect ordinary immediate memory, where a set of material is presented to one sensory modality or channel (Brown, 1954; Conrad, 1957; Peterson \& Peterson, 1959; Wickelgren, 1965) and dichotic memory, where different materials were presented to the two ears simultaneously (Broadbent, 1954, 1956, 1957b, 1958). No study, however, has considered all of them concurrently or in bisensory memory so as to evaluate their interactive roles. The present study examines each of these variables in a factorial design permitting an assessment of their effects on visual and auditory modalities under conditions of simultaneous stimulation.

Of particular interest to the present study were modality comparisons. Reviewing the literature, it was found that a distinction between the visual and auditory modalities in terms of effects of the foregoing variables did not exist. Rather, the general trend has been to assume that effects observed with an auditory presentation would also obtain for visual presentation. More precisely, there has been a lack of concern for specific visual effects. Experiments employing the visual modality have not considered modality to be a pertinent condition of the experinent. Mackworth's study (1962) of visual rate effects is among the very first to suggest that the auditory and visual channels may function differently in respect to the foregoing variables and particularly rate of presentation.

\section{Apparatus}

\section{METHOD}

Materials were presented on $16 \mathrm{~mm}$ films ( 24 frames/sec) with synchronous sound track by means of an RCA projector. Speech recordings were spliced into the film so that each utterance of a stimulus item commenced within the first half frame of its associated visual stimulus ( $\pm 21 \mathrm{msec}$ ).

Visual stimuli were made by "Letraset" instant black letters exposed singly on white $8 \frac{1}{2}$ in. $\times 11$ in. cards which were then filmed and appropriately edited. The interstimulus and interlist intervals for visually presented materials consisted of clear leader which was slightly less bright than the stimulus sequence thus minimizing the negative after-image.

An attempt was made to balance the intensity of the materials in the two channels on reproduction by subjective equality judgments by five Ss. However, it seemed reasonable to assume that the intensities might not be the crucial variable so long as the material was clearly and easily discriminable.

The experiment was performed in a large lecture hall and Ss were centrally seated.

\section{List Preparation and Presentation}

The first 10 letters of the alphabet, A through J, were employed as were the digits 0 through 9. Pilot studies measuring tachistoscopic thresholds of the 26 letters of the alphabet indicated that each item was recognized with equal facility.

Each list contained six items. Altogether 48 lists were required in order to produce 24 pairs of lists sampling all possible combinations of three rates of presentation. Two orders of report, and four combinations of materials. The material combinations were: (1) auditory-numbers, visual-numbers, (2) auditorynumbers, visual-letters, (3) auditory-letters, visual-letters, and (4) auditory-letters, visual-numbers. Where items had to be employed twice this was done within rather than across modalities.

Visual items were presented for $.5 \mathrm{sec}(12 \mathrm{frames})$; the duration of the auditory items averaged 10 frames or $.42 \mathrm{sec}$. Materials were presented at three rates: (1)1 pr/.5 sec, no interstimulus interval (fast); (2) $1 \mathrm{pr} / \mathrm{sec}, .5 \mathrm{sec}$ interstimulus interval (medium); (3) 1 $\mathrm{pr} / 2 \mathrm{sec}, 1.5 \mathrm{sec}$ interstimulus interval (slow). There was a $20 \mathrm{sec}$ interlist interval, with $15 \mathrm{sec}$ for recall and a ready signal given 5 sec before the start of each list.

\section{Subjects}

Sixty volunteer undergraduate introductory psychology students served as Ss and were tested in groups of 10.

\section{Administration}

Ss were divided into two instructional groups: half of them were pre-instructed regarding order of report and half of them were post-instructed. Each group of $10 \mathrm{Ss}$ was assigned to one of the instructional groups. Within the two instructional groups, rate of presentation, materials interaction, and order of report were varied. The same lists were used for both instructional groups. The order of presentation of the rate variable was partially counterbalanced in Latin-square manner. That is, $10 \mathrm{Ss}$ in each instructional group received the $F, M, S$ order; 10 received the $M, S, F$ order; and the remaining 10 received the $S, F, M$ order. Separate films, each duplicated from the original, were used for each order of rate presentation. On the films presented to the pre-instructed group the word "auditory" or "visual," indicating the prescribed order of report, was spoken immediately after the ready signal and 


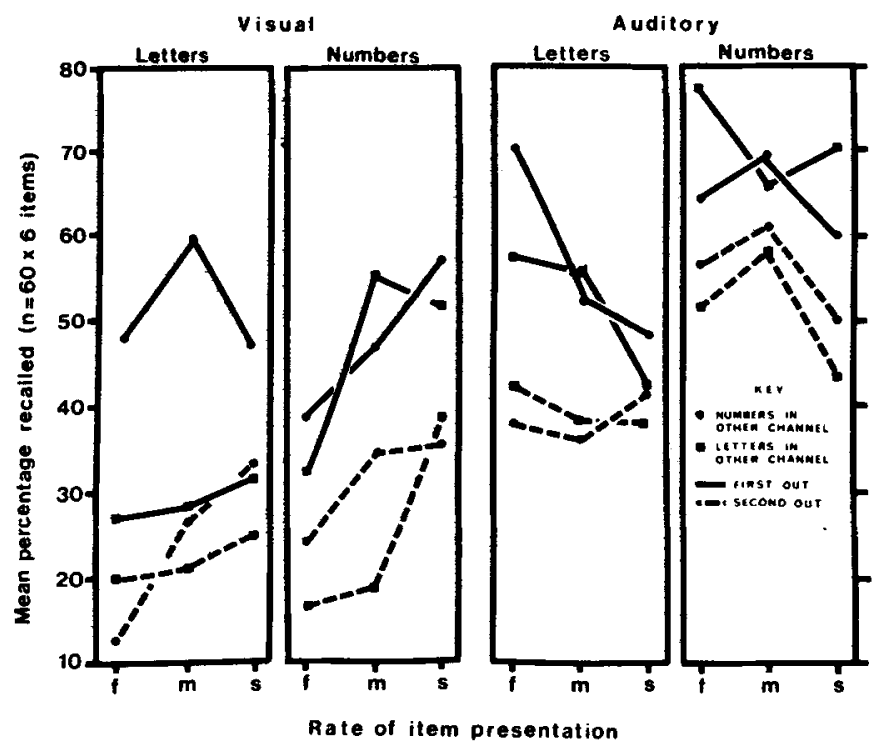

Fig. 1. Graphic representation of data scored on item-by-item basis (expressed in percentages)

before the presentation of the list. For the post-instructed group these terms were given immediately after the presentation of the last item in the list. As indicated, half of the lists were preceded by or followed by "auditory" and half by "visual" in a pre-arranged random order.

A written response was required of $S$. Instructions emphasized serial reproduction and attention to both sets of stimuli.

\section{RESULTS}

The data were scored according to the accuracy of responses both in nature and location, the data for each $\mathrm{S}$ being scored on an item-by-item basis permitting individual scores to take on values between $0-6$.

The average scores for each of the 48 tasks are shown in Fig. 1, and are expressed as percentages.

The following relations among the variables may be discerned in this figure: more items are retained from the channel reported first than from the channel reported second; numbers are generally retained better than letters; auditory retrieval is best at the fastest rate while visual retrieval is best at the slowest rate. Also, the nature of the material in the opposing channel appears to be relevant only for visual letters.

To render these observations more precise and to determine the relative importance of each stimulus dimension, the performances of each $\mathbf{S}$ were summed in all possible ways. The mean performances associated with each stimulus dimension are shown in Table 1 where the significance tests appropriate to each dimension are indicated. It is observed that differences in each dimension had a significant effect on the number of items reported.

The data were next examined for the purpose of determining interactions among the stimulus dimensions. To this end, the possibility was considered of performing a five-dimensional analysis-of-variance, but the idea was dismissed for two reasons. In the first place, it was certain that high-order interactions would emerge from any idiosyncratic features of any of the 48 strings, since each of them represented a unique combination of the materials, rate, modality, and order dimensions. In the second place, it was apparent from Fig. 1 that the nature of the material in the other channel on any task interacted with performance in an asymetric fashion.

For these reasons each dimension was intercompared with every other dimension by means of two-way analyses-of-variance. Only three of 10 possible interactions proved to be significant. These were: (1) modality and materials $(F=24.27, \mathrm{df}=1 / 59, \mathrm{p}<.001)-$ although letters are retrieved more poorly than numbers, the disparity is much greater in the auditory channel than it is in the visual channel. A Scheffé test for multiple comparisons established titat each of the four combinations of modality-materials differed significantly from one another $(p<.001) ;(2)$ modality and rate $(\mathrm{F}=22.46, \mathrm{~d} \mathbf{f}=2 / 118, \mathrm{p}<.001)$-as rate decreases, auditory performance lessens and visual performance improves. Indeed progressions of means are quite regular-for auditory they are 4.6-4.4-4.0, and for vision they are $2.1-2.9-3.2$, in fastmedium-slow order. Scheffé tests for these data established that auditory retrieval at the slow rate and visual retrieval at the fast rate were significantly poorer than at their other rates $(p<.001)$; (3) instructions and order of report $(\mathrm{F}=5.79, \mathrm{df}=1 / 58, \mathrm{p}<.001-$ the nature of the interaction is that pre-instruction emphasizes the disparity between the channel reported first and the channel reported second. The means for the pre-instructed group are $3.5-2.2$, and for the post-instructed group are 2.8-2.1, in first out-second out order. $T$ tests established that the difference between the pre- and post-instructed group remained highly significant for the channel reported second.

Finally, the data for each modality-materials combinations were examined in terms of the context in which they occurred. Posed as a question, did the nature of the material occurring simultaneously in the other channel affect the success with which items were reported? Since a significant interaction was observed between modality and material, the question was examined separately for each modality-material combination. The results are shown in Table 2. Recall scores for like and unlike accompaniments indicates that for three of the combinations, related sample t-tests indicate that performance is independent of the nature of the

Table 1

Means and (Standard Deviations) Associated with each Dimension of Bisensory Stimulation*

(maximum score $=6$ )

\begin{tabular}{lllllllllll} 
& & & & & & DF & test & P \\
\hline Overall: & $2.65(.61)$ & & & & & & & & \\
Order: & First: & 3.14 & $(.90)$ & Second: & 2.16 & $(.65)$ & 59 & $\mathrm{t}=8.11$ & .001 \\
Modality: & Auditory: & 3.24 & $(.88)$ & Visual: & 2.06 & $(.64)$ & 59 & $t=10.57$ & .001 \\
Materials: & Numbers: & 2.97 & $(.68)$ & Letters: & 2.33 & $(.61)$ & 59 & $t=11.59$ & .001 \\
Instructions: & Pre: & 2.89 & $(.60)$ & Post: & 2.41 & $(.51)$ & 58 & $t=4.68$ & .001 \\
Rate: & Fast 2.52 (.68) & & Medium 2.74 (.66) & Slow 2.69 (.67) 2.118 & $\mathrm{F}=4.06$ & .05 \\
\hline
\end{tabular}

"A fixed effects model is assumed for this analysis and succeeding analyses in which the individual stimulus items are presumed to have equal diaficulty. In partial corroboration, the variances associated with each of the 24 stimulus strings reported out first were intercompared and an F-ratio between the largest and smallest variance failed to achieve significance. It remains possible, however, that some of the variance associated with experimental treatments ought properly to be assigned to the stimulus strings themselves. In this case all errors terms would be inflated and the present high significance levels would be somewhat diminished. 
Table 2

Mean Context Responses and (Standard Deviations)

for the Item Analysis

(DF = 59)

Accompaniment

\begin{tabular}{lcccc} 
Modality-Material & Like & Unlike & t & P \\
\hline Numbers-Auditory & $3.69(1.09)$ & $3.74(.96)$ & .013 & - \\
Letter-Auditory & $2.73(.99)$ & $2.76(1.00)$ & .009 & - \\
Numbers-Visual & $2.32(.96)$ & $2.09(.74)$ & 1.920 & - \\
Letters-Visual & $1.56(.71)$ & $2.29(.91)$ & 5.330 & .001 \\
\hline
\end{tabular}

material in the other channel. For the fourth combination, visual letters, however, a highly significant disparity obtains. Thus, retrieval of visual letters appears to be suppressed by the concurrent presentation of letters in the auditory channel.

\section{DISCUSSION}

Several of the variables studied produced effects in bisensory memory similar to those recorded for them in the existing literature on unisensory memory. For example, it was not unexpected that the channel reported first should be the channel recalled best, simply because it is first out, and therefore not subject to lengthy storage demands. That instructions facilitated overall bisensory recall is also in keeping with the bulk of data collected on the effect of preparatory set.

There were also material differences which were not without a precedent. Thus, while the letters variable was defined by ten items, these ten items were part of a potentially larger response set consisting of all 26 letters of the alphabet. There is no assurance that instructions restricting the stimulus set served to adequately restrict Ss' response set (Garner, 1962). By contrast, the 10 digits defined both the stimulus and response set. Therefore it is not surprising that numbers prove considerably easier to recall than letters under any conditions of short-term memory.

Thus the variables of instruction, order of report, and nature of materials yielded effects in the bisensory situation similar to those reported for the dichotic and ordinary immediate memory situations. On the other hand, there were variables that yielded results which were unexpected on the basis of existing literature. More precisely, it was not clear in advance how these variables would influence bisensory memory. Thus the modality-rate interaction, i.e, differences in amount recalled in each modality at each rate, provides the most intriguing effect and as such will be discussed in some detail as will the materials-modality effect.

\section{Modality-rate Interaction}

Bisensorily, both modalities displayed curves predictable from data obtained for each when it was explored independently of the other modality (Dornbush, 1967a, 1967b). Thus the integrity of the modality-rate results was maintained even when both modalities were stimulated simultaneously. Auditory results yielded the typically obtained decay function with decreasing rate. That this independence of modality was maintained was most surprising particularly in view of results obtained for the only other condition of simultaneous stimulation given previous attention, the dichotic situation. Briefly, in the latter case, recall was better at slower rather than faster rates, presumably because of the use of two channels.

In the visual modality, the finding that slower rates yield better recall even though this result has been found previously (Mackworth, 1965) requires an explanation, since decay theory in its present form cannot predict this outcome.

The nature of the rehearsal process provides a plausible basis for explaining the visual data and also the contrasts between visual and auditory recall. Broadbent (1963), Sperling (1960), and Wickelgren (1965) have expressed the view that both auditory and visual stimuli are transformed into auditory subvocalizations. Wickelgren has further suggested that short-term storage, even when materials are presented visually, is auditory (speech-motor). Finally, Sperling (1963) proposes, as a model for visual recall, that a visual stimulus is first stored in VIS, visual information storage, in the form of a visual image. It is then scanned and rehearsed. The rehearsal component produces a verbal response "audible" in AIS, auditory information storage, or a vocal response audible to another person and indirectly also in AIS.

This alleged necessity to transpose incoming information from visual input to an aurally-based storage may serve to retard visual recall, particularly at the fastest rates. Required for this alternation may be an interval of time long enough to affect the processing of materials arriving at a very rapid rate. By contrast, material on the auditory channel may be "shadowed" subvocally almost in simultaneity with its arrival.

If this is the case, at the fastest rate, $S$ may rehearse all of the items in auditory input first, since switching from one modality to the other at this rate might not be the most economical method of rehearsal. This is what was suggested by Broadbent (1956), who found, in a dichotic situation, that Ss recall all of the material from one ear first and then from the other. With a slow delivery rate, however, he found recall to be possible in the order of arrival or pair-by-pair. Broadbent reasoned that at the faster rates $S$ was not able to switch from ear to ear fast enough to make possible order of arrival reports. In the present situation, if all the auditory material is processed first, as suggested, $S$ has the difficult task of recognizing and rehearsing items from the fading trace of visual input. That this is what occurs is also put forth as a possible explanation of visual results by Posner (1967). "The idea of an auditory information storage system suggests that storage of information presented aurally is subject to simpler encoding than visually presented information . . . Since encoding or acquisition strategies require time, this indicates that visual storage involves more of such strategies [p.275]"

However, while it is assumed that visual input enters an auditory store the characteristics of the visual curve indicate that it may be a store somewhat different from the store to which items that are initially auditory terminate (Dornbush, 1967b; Margrain, 1967).

\section{Material Interactions}

It was demonstrated that it does not matter whether the accompaniment to a given stimulus is composed of similar or dissimilar materials except in the case of visual letters. That is, how successfully auditory materials are recalled is irrelevant of what is going on in the visual modality. The same is true for the visual modality but only when numbers are on this channel; i. e., similar number of lists are recalled when either type of material opposes numbers on the visual channel. However, when letters are on the visual channel, this channel is explicably jammed when letters are also on the opposing or auditory channel. That acoustic similarity between the materials on both channels is not necessarily responsible for this jamming is suggested by the fact that decrement occurs only for recall of visual letters when accompanied by auditory letters but no decrement occurs for the reverse combination, i.e., a recall of auditory letters when accompanied by visual letters. Posner (1967) indicates that while it has been difficult to demonstrate retroactive interference effects based on similarity, a more appropriate division to demonstrate such effects has been acoustic confusability. The present experiment indicates that even this division may be subject to retroactive effects under limited conditions only.

The foregoing results lead to the conclusion that an individual cannot successfully do two things at once. That is, $S$ cannot attend to both channels equally-or if he can attend to both channels equally, he cannot recall them equally well. Several procedures are being introduced to visual presentation in an attempt to equate visual and auditory recall. Under investigation now is the effect of "shadowing" (vocalization-at-presentation) one of the modalities. Specifically, this procedure should result in simultaneous trunslation of the visual items if, in fact, subvocalization or translution is relevant. 


\section{REFERENCES}

BROADBENT, D. E. The role of auditory localization in attention and memory span.J. exp. Psychol, 1954, 47, 191-196.

BROADBENT, D. E. Successive responses to simultaneous stimuli. Quart. $J$. exp. Psychol, 1956, 8, 145-152.

BROADBENT, D. E. Immediate memory and simultaneous stimuli. Quart. J. exp. Psychol., 1957b, 9, 1-11 (b).

BROADBENT, D. E. Perception and communication. London: Pergamon Press, 1958

BROADBENT, D. E. Flow of information within the organism. $J$. verbal Learn, verbal Behav., 1963, 2, 34-39.

BROWN, J. The nature of set-to-learn and of intra-material interference in immediate memory. Quart. J. exp. Psychol, 1954, 6, 141-148.

CONRAD, R. Decay theory of immediate memory. Nature, 1957, 179 , 831-832.

DORNBUSH, R. L. Shadowing in bisensory memory. Quart. J. exp. Psychol, $1967 \mathrm{a}$

DORNBUSH, R. L. Input variables in bisensory memory. Unpublished doctoral dissertation. City University of New York, $1967 \mathrm{~b}$.

GARNER, W. R. Uncertainty and structure as psychological concepts. New York: John Wiley \& Sons, 1962.

MACKWORTH, J. F. Presentation rate and immediate memory. Canad. $J$. Psychol, 1962, 16, 43-47.

MARGRAIN, S. A. Short-term memory as a function of input modality. Quart. J. exp. Psychol, 1967, 109-114.

MORAY, N. Broadbent's filter theory:postulate $H$ and the problem of switching time. Quart. J. exp. Psychol, 1960, 12, 214-220.

PETERSON, L. R., \& PETERSON, M. J. Short-term retention of individual verbal items. $J$. exp. Psychol., 1959, 58, 193-198.

POSNER, M. I. Short-term memory systems in human information processing. In A. F. Sanders (Ed.), Attention and performance. Amsterdam: North-Holland Publishing Co., 1967.

SPERLING, G. The information available in brief visual presentations. Psychol. Monogr., 1960, 74, No. 11 (Whole No. 498).

SPERLING, G. A model for visual memory tasks. Hum. fact., 1963, 5, 19-31. WICKELGREN, W. A. Acoustic similarity and intrusion errors in short-term memory.J. exp. PsychoL, 1965, 70, 102408.

\section{NOTES}

1. Based upon a dissertation submitted to the Graduate Faculty in Psychology of the City University of New York in partial fulfillment of the requirements for the degree of Doctor of Philosophy. The author acknowledges the guidance of Professors Wilma A. Winnick and Lawrence R. Zeitlin, and in particular, the invaluable encouragement, support and direction of Professor Louis J. Gerstman.

2. Now at Department of Psychiatry, New York Medical College, FlowerFifth Avenue Hospitals, New York, N.Y.

3. A considerably abbreviated form of this research and different data analysis appears in the Proceedings of the XVIIIth International Congress of Psychology, Moscow, USSR, 1966.

(Accepted for publication March 4, 1968.) 